This item was submitted to Loughborough's Research Repository by the author.

Items in Figshare are protected by copyright, with all rights reserved, unless otherwise indicated.

\title{
Multiscale modeling for the heterogeneous strength of biodegradable polyesters
}

PLEASE CITE THE PUBLISHED VERSION

https://doi.org/10.1016/j.jmbbm.2018.10.018

\section{PUBLISHER}

(C) Elsevier

VERSION

AM (Accepted Manuscript)

\section{PUBLISHER STATEMENT}

This paper was accepted for publication in the journal Journal of the Mechanical Behavior of Biomedical Materials and the definitive published version is available at https://doi.org/10.1016/j.jmbbm.2018.10.018

LICENCE

CC BY-NC-ND 4.0

\section{REPOSITORY RECORD}

Zhang, Taohong, Geyu Jin, Xiaoxiao Han, Yue Gao, Qingfeng Zeng, Binbin Hou, and Dezheng Zhang. 2019. "Multiscale Modeling for the Heterogeneous Strength of Biodegradable Polyesters". figshare. https://hdl.handle.net/2134/35627. 


\section{Author's Accepted Manuscript}

Multiscale Modeling for the Heterogeneous Strength of Biodegradable Polyesters

Taohong Zhang, Geyu Jin, Xiaoxiao Han, Yue Gao, Qingfeng Zeng, Binbin Hou, Dezheng Zhang

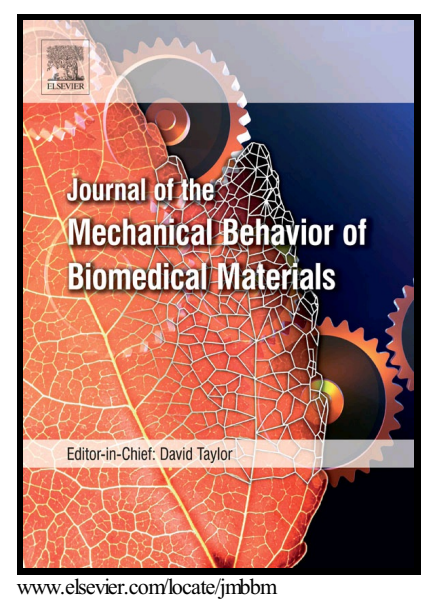

PII: $\quad$ S1751-6161(18)30874-9

DOI: $\quad$ https://doi.org/10.1016/j.jmbbm.2018.10.018

Reference: JMBBM3032

To appear in: Journal of the Mechanical Behavior of Biomedical Materials

Received date: 6 June 2018

Revised date: 28 August 2018

Accepted date: 9 October 2018

Cite this article as: Taohong Zhang, Geyu Jin, Xiaoxiao Han, Yue Gao, Qingfeng Zeng, Binbin Hou and Dezheng Zhang, Multiscale Modeling for the Heterogeneous Strength of Biodegradable Polyesters, Journal of the Mechanical Behavior of Biomedical Materials, https://doi.org/10.1016/j.jmbbm.2018.10.018

This is a PDF file of an unedited manuscript that has been accepted for publication. As a service to our customers we are providing this early version of the manuscript. The manuscript will undergo copyediting, typesetting, and review of the resulting galley proof before it is published in its final citable form. Please note that during the production process errors may be discovered which could affect the content, and all legal disclaimers that apply to the journal pertain. 


\title{
Multiscale Modeling for the Heterogeneous Strength of Biode-
}

\section{gradable Polyesters}

\author{
Taohong Zhang ${ }^{1,2^{*}}$ Geyu Jin ${ }^{1,2}$ Xiaoxiao $\mathrm{Han}^{3}$ Yue Gao ${ }^{1,2}$ Qingfeng Zeng ${ }^{1,2}$ Binbin Hou ${ }^{1}$ \\ Dezheng Zhang ${ }^{1,2}$
}

${ }^{1}$ Department of Computer, School of Computer and Communication Engineering, University of Science and Technology Beijing (USTB), Beijing, 100083, China.

${ }^{2}$ Beijing Key Laboratory of Knowledge Engineering for Materials Science, Beijing, 100083, China.

${ }^{3}$ Wolfson School of Mechanical, Electrical and Manufacturing Engineering, Loughborough University, Loughborough, Leicestershire, LE11 3TU, UK.

\begin{abstract}
A heterogeneous method of coupled multiscale strength model is presented in this paper for calculating the strength of medical polyesters such as polylactide (PLA), polyglycolide (PGA) and their copolymers during degradation by bulk erosion. The macroscopic device is discretized into an array of mesoscopic cells. A polymer chain is assumed to stay in one cell. With the polymer chain scission, it is found that the molecular weight, chain recrystallization induced by polymer chain scissions, and the cavities formation due to polymer cell collapse play different roles in the composition of mechanical strength of the polymer. Therefore, three types of strength phases were proposed to display the heterogeneous strength structures and to represent different strength contribution to polymers, which are amorphous phase, crystallinity phase and strength vacancy phase, respectively. The strength of the amorphous phase is related to the molecular weight; strength of the crystallinity phase is related to molecular weight and degree of crystallization; and the strength vacancy phase has negligible strength. The vacancy strength phase includes not only the cells with cavity status but also those with an amorphous status, but a molecular weight value below a threshold molecular weight. This heterogeneous strength model is coupled with micro chain scission, chain recrystallization and a macro oligomer diffusion equation to form a multiscale strength model which can simulate the strength phase evolution, cells status evolution, molecular weight, degree of crystallinity, weight loss and device strength during degradation. Different example cases are used to verify this model. The results demonstrate a good fit to experimental data.
\end{abstract}

Keywords: Heterogeneous method; Multiscale modeling; Strength modeling; Degradable Polyesters; Mathematical Simulation

\begin{tabular}{|llll|}
\hline Nomenclature & $\varepsilon$ & $\begin{array}{l}\text { porosity of polymer device due to hydrolysis } \\
\text { reaction }\end{array}$ \\
$M_{\mathrm{n} 0}$ & initial polymer molecular weight & $\vartheta$ & $\begin{array}{l}\text { threshold degree of small molecules solution } \\
\bar{M}_{\mathrm{n}}\end{array}$ \\
normalized average molecular weight & $\pi_{v 1}$ & $\begin{array}{l}\text { rate constant of the uncatalytic hydrolysis reac- } \\
\text { tions of ester bonds } v\end{array}$ \\
$R_{\mathrm{s}}$ & the total scission chains & $\pi_{v 2}$ & rate constant of the autocatalytic hydrolysis
\end{tabular}




\begin{tabular}{|c|c|c|c|}
\hline \multicolumn{4}{|c|}{ ACCEPTED MANUSCRPT } \\
\hline$p$ & the probability for recrystallization & $X_{\mathrm{A}}(t)$ & $\begin{array}{l}\text { reactions of ester bonds } v \\
\text { Volume ratio of amorphous strength phases at } \\
\text { time } t\end{array}$ \\
\hline$N_{c}$ & crystallization chains numbers & $X_{c}(t)$ & degree of crystallinity at time $t$ \\
\hline$x(i, j, t)$ & cellular $(i, j)$ status at time $t$ & $R_{\mathrm{ol}}$ & oligomers produced by chains scission \\
\hline$M_{n}(i, j, t)$ & cellular $(i, j)$ molecular weight at time $t$ & $m$ & threshold degree of oligomers \\
\hline$N(i, j, t)$ & cellular $(i, j)$ chains at time $t$ & $n$ & cellular grid number \\
\hline$t_{s}$ & chain scission iteration time & $t$ & degradation time \\
\hline$\Delta t$ & chain scission time step length & $\Delta t_{d}$ & oligomers diffusion time step length \\
\hline$\alpha_{1}$ & $\begin{array}{l}\text { the coefficient in strength model of } \\
\text { amorphous phase }\end{array}$ & $B_{1}$ & $\begin{array}{l}\text { the exponent in strength model of amorphous } \\
\text { phase }\end{array}$ \\
\hline$\alpha_{2}$ & $\begin{array}{l}\text { the coefficient in strength model of crys- } \\
\text { talline phase }\end{array}$ & $B_{2}$ & $\begin{array}{l}\text { the exponent in strength model of crystalline } \\
\text { phase }\end{array}$ \\
\hline$D$ & $\begin{array}{l}\text { diffusion coefficient of oligomers in de- } \\
\text { graded polymers }\end{array}$ & $D_{0}$ & $\begin{array}{l}\text { oligomers diffusion coefficient in polymer } \\
\text { amorphous district }\end{array}$ \\
\hline$M_{n \mathrm{C}}^{\sigma}$ & $\begin{array}{l}\text { threshold molecular weight for judging } \\
\text { whether the cell strength is zero }\end{array}$ & $D_{\varepsilon}$ & $\begin{array}{l}\text { diffusion coefficient of oligomers in liquid-filled } \\
\text { pores }\end{array}$ \\
\hline$\sigma_{\mathrm{C}}$ & Strength of crystalline phase & $M_{\mathrm{nc}}$ & $\begin{array}{l}\text { threshold molecular weight for judging whether } \\
\text { the cell's status become hole (-1) or not }\end{array}$ \\
\hline $\bar{\sigma}$ & normalized device strength & $\sigma_{\mathrm{A}}$ & Strength of amorphous phase \\
\hline
\end{tabular}

\section{Introduction}

Biodegradable polymers, such as polylactide (PLA), polyglycolide (PGA) and their copolymers are attracting more and more attention in the fields of orthopaedics, tissue engineering, and drug delivery [1-4]. The biodegradable devices provide sufficient mechanical support at the beginning, and then during the degradation process, the strength decreases gradually to allow a gradual transfer of loads to regenerated tissues. With appropriate mechanical stimulations, tissues can be fully recovered. Therefore, controlling the rate of strength change during degradation is one of the main challenges [5]. There are many methods that can be used to address the challenge, which include altering polymer properties (such as initial molecular weight and initial crystallinity) and changing the ratio of copolymers. To understand how the strength is evolving during degradation is the basis for selecting appropriate methods to address the problem.

Some experimental studies have been carried out to reveal the strength evolution during degradation [6-9]. However, it is difficult to predict the strength during degradation using the data collected from multiple different sample geometries because PLA, PGA and their copolymers undergo both non-catalytic and autocatalytic hydrolysis reactions. Autocatalytic hydrolysis reaction leads to size effect [10]: degradation rate in the centre of a device is higher than that around the boundaries. Additionally, cavities are generated in the centre of the device which lead to further strength degeneration. Recrystallization induced by the hydrolysis chain scission [11] makes polymer stiffer and stronger. These make the strength change during the degradation process 


\section{ACCEPTED MANUSCRIPT}

more complicated. The experimental method is laborious and time-consuming. Mathematical models can help to understand the degradation process and provide quantitative data during the whole degradation process for optimal device design. Pan and coworkers proposed models of Young's modulus change of biodegradable polymers during biodegradation, ranging from an entropy spring model, an atomic finite element model to a constitutive law for degrading bioresorbable polymers during degradation [12-14]. Shirazi et al. [15] proposed a coupled model (coupling of elastic properties model and molecular weight model developed by Wang et al. [16]) to simulate the mechanical behaviour of polymers during degradation. A non-linear viscoelastic model to describe the evolution of mechanical behaviour of polymers during the hydrolytic degradation was developed by Breche et al. $[17,18]$. These modelling methods focus on Young's modulus instead of strength. Flory [19] put forward a modelling formula $T=a_{0}+a_{1} / M$ to describe the relationship of polymer's strength $(T)$ and number average molecular weight $(M)$, in which $a_{0}, a_{1}$ are the material-specific coefficients. This formula is a simplified mathematical fitting, which is not suitable for fitting the complicated polymer degradation process. Up to the present, there are few research studies on strength evolution of biodegradable polymers during degradation.

In this paper, a heterogeneous multiscale method is proposed to simulate the tensile strength change caused by micro chain scission, recrystallization, macro oligomers diffusion and cavities appearance. The influence of molecular weight and the degree of crystallization on tensile strength change are analyzed as the major factors. While smaller molecular weight reduces the strength, crystallization makes polymer stiffer and stronger. On the other hand, both cavities that caused by oligomer diffusion and small oligomers with molecular weight lower than a critical value contribute little to strength. Three different strength phases are introduced to the heterogeneous structure: amorphous phase, crystallization phase and strength vacancy phase. The strength vacancy phase is used to distinguish from cavity shapes which are generated by oligomers diffusion or dissolution of small molecules. The strength vacancy phase threshold molecular weight is obtained in different case studies. The computation process including molecular weight distribution, strength phase evolution and distribution, chain number, the degree of crystallinity, the evolution of polymer inner shape and weight loss during degradation can be obtained from the heterogeneous strength model simultaneously. The computation results in the demonstration examples show that the model can fit experimental data in the literature very well.

\section{A Heterogeneous Strength Modelling Method for Polymers Degradation}

\subsection{Major Factors Influencing Strength Change during Degradation}

The mechanism of tensile strength change during the degradation of biomedical polymers is not clear yet. According to the experimental research, the degeneration tendency of polymer mechanical properties is quite similar to that of molecular weight reduction, which means the change of polymers tensile strength during the degradation, to a great extent, depends on the decrease of molecular weight $[20,21]$. Shorter polymer chains wind less firmly and less complicatedly, and the polymer twine is easier to be unfastened, which will make the strength decreasing. The chain scission reaction produces short polymer chains. And the cleavages of chains also result in the reduction of molecular weight. This is the reason that the degenerating trends of 


\section{ACCEPTED MANUSCRIPT}

tensile strength and molecular weight are similar.

The degree of crystallization also has a significant effect on the change of tensile strength. It is believed that crystallization leads to an increase of polymer strength because the polymer chains are arranged more closely and more strongly bonded to each other in the crystallized area, which makes them more resistant to deforming. Therefore the stiffness of crystallised polymer is much higher than amorphous polymer, and a similar trend is expected for the tensile strength.

In Saha and Tsuji's experiment about the mechanical properties of poly(I-lactide-co- $\varepsilon$ - caprolactone) during the degradation [22], the effects of molecular weight and the degree of crystallization on the tensile strength change are clearly demonstrated. In the early stage of degradation, the polymer chains have a high probability to crystallise and the degree of crystallization increases sharply. The polymer becomes stiffer and stronger even though molecular weight reduces due to chain scission. During this period, the contribution of crystallinity to strength is higher than the effect of molecular weight on strength. However, with increasing degradation time, the reduction of molecular weight plays an increasingly significant role in the change of strength. In the later stage of degradation, molecular weight drops to a very low value, holes appear in the polymer, and strength is lost. In another experiment, samples are stored in a dry environment. Therefore, the hydrolysis reaction, which requires the presence of water, cannot occur and molecular weight remains constant. However, crystallinity still varies and affects strength. This experiment shows the strength increases and then remains constant due to the influence of degree of crystallization, as opposed to reducing (as seen when the hydrolysis reaction occurs).

\subsection{Heterogeneous Strength Model of Polyesters during Degradation}

We propose a multiscale heterogeneous strength model to reveal the strength change during the degradation process. In this section, we will discuss the definition of different phases in the mesoscopic-scale, and the integration of three scales to characterize the multiscale heterogeneous strength model.

The following assumptions are adopted in this paper on the process of polyester degradation:

(i) amorphous polymer chains can hydrolyse, but are too large to diffuse;

(ii) crystalline phase can neither hydrolyse nor diffuse;

(iii) oligomers catalyse the hydrolysis reaction, are soluble in the polymer matrix and can diffuse out of a polymer device;

(iv) water diffusion is fast such that water molecules are abundant

The macroscopic device is discretized as $n \times n$ mesoscopic cells. It is assumed that each cellular lattice has one polymer chain, which undergoes hydrolysis reaction. The micro-scale model is designed to simulate the polymer chain scission and recrystallization. In the hydrolysis reaction, water molecules attack the polymer chains and cause their breakage into shorter ones. The chain scission produces oligomers which generate an acidic environment and lead to a faster hydrolysis reaction, i.e. the reaction is autocatalytic. The phenomenon of autocatalysis is a significant feature in bulk erosion. For modelling polymer chain scission, the chemical reaction can be described as:

$$
\mathrm{P}_{2}+\mathrm{W} \rightarrow 2 \mathrm{P}_{1}
$$




$$
\begin{aligned}
& \mathrm{P}_{3}+\mathrm{W} \rightarrow \mathrm{P}_{1}+\mathrm{P}_{2} \\
& \quad \ldots \\
& \mathrm{P}_{M}+\mathrm{W} \rightarrow \mathrm{P}_{M-r}+\mathrm{P}_{r}(r=1,2, \ldots, M-1)
\end{aligned}
$$

Here $W$ stands for water molecules, $P_{2}, P_{3}, \ldots$, and $P_{M}$ stand for polymer chains with $2,3, \ldots$, and $M$ ester bonds. In the computer simulation, the location and time at which each chain cleavage occurs should be set. The kinetic Monte Carlo (MC) method is used to determine the cleavage chain and the time $[21,23]$. Following the MC scheme, the chain scission location, ester bond $\mu$ (with $\mu$ ester bonds) is selected for chain breakage according to the following:

$$
\sum_{V=1}^{\mu-1} \alpha_{V}<r_{2} \sum_{V=1}^{M} \alpha_{V} \leq \sum_{V=1}^{\mu} \alpha_{V}
$$

with the following length of time integration step:

$$
\Delta t=\frac{1}{\sum_{v=1}^{M} \alpha_{v}} \ln \left(\frac{1}{r_{1}}\right)
$$

in which $r_{1}, r_{2}$ are two random numbers ranged from $(0,1]$ to simulate the stochastic nature of the reaction, $v$ is a natural number, $\sum_{v=1}^{M} \alpha_{v}$ stands for the production of all the possibility of chain scissions which include both uncatalytic and autocatalytic hydrolysis reactions and $M$ is the biggest ester bond number of the longest polymer chain. Following ref [21] the molecule degree is given by

$$
\alpha_{v}=\pi_{v 1} X_{\mathrm{p} v} X_{\mathrm{W}}+\pi_{v 2} X_{\mathrm{p} v} X_{\mathrm{ol}}^{0.5} X_{\mathrm{W}} \quad(v=1,2, \cdots, M)
$$

in which $X_{\mathrm{P} v}, X_{\mathrm{ol}}, X_{\mathrm{W}}$ are respectively the reactant molecules (polymer with the ester bond unit $v$, oligomers and water molecules). $\pi_{v 1}, \pi_{v 2}$ are the rate constants for the uncatalytic and autocatalytic hydrolysis reactions of ester bonds $v$. The numerical value of the dimensionless rate constants are the same as the reactions constants $k_{1}$ and $k_{2}$ in Ref.[21]. The autocatalytic reaction is judged by whether oligomers exist or not. The chain broken pattern is used as the result of whether autocatalytic reaction or not. If there are oligomers existing in cell or neighbor cells, the random chain scission happens; otherwise, the end chain scission is chosen as the chain broken pattern. It is further assumed that the crystallized polymer chains do not undergo hydrolysis because it takes a long time for water to penetrate these nano-crystals. Using $R_{S}$ to represent the total number of chain scissions, following Gleadall et al. [11] the degree of chain cleavage induced crystallization, crystallization chains numbers is denoted as $N_{c}$, which can be calculated as a probability of chain scissions:

$$
N_{c}=p R_{S}
$$

in which $p$ represents the probability of recrystallization. Eq. (4) is represented for the recrystallization induced by chain scission.

With chain scissions, recrystallization and cavities appear during the degradation, the inner 


\section{ACCEPTED MANUSCRIPT}

material structure becomes heterogeneous. Different cellular status are proposed here to represent the evolution of cellular structure. There are four statuses occur for a cell evolution. At the beginning, the cellular status can be either amorphous or crystalline. Once the chain scission occurs, the status of this cell is changed to scission status. When a hole appeared in the cellular grid, the status is referred to as hole status. These statuses can be represented by digits:

$$
x(i, j, t)= \begin{cases}1 & \text { initially amorphous } \\ 0 & \text { chain scission occurred in cell } \\ -1 & \text { hole } \\ -2 & \text { crystallized }\end{cases}
$$

And the cellular status evolution rules are as following:

i. The initial amorphous cellular status are set as 1 , and the initial crystallized cellular status is set as -2 ; The status of crystallized cells will not be changed from -2 to 0 or -1 ;

ii. Once a chain scission occurs, the status of this cell is changed to 0 ;

iii. If more than $90 \%$ of the chains crystallized in a cell, the status of this cell is changed to -2 ;

iv. If the molecular weight decreases to a threshold molecular weight $M_{\mathrm{nc}}$ the status of this cell is changed to -1 , which indicates the cell has become a hole.

With the cellular structure status evolution, the chain scission and recrystallization of each cell affect strength change in different ways. Another impact factor is the pore appearance. It is assumed that there is no strength in the pore zones. While from the fitting calculation, it is found that when the molecular weight reduces to a threshold value, there is not much strength sustained in the cell.

Based on the three different zones, heterogeneous strength model is proposed in each cell as follows:

1) The amorphous strength phase

If there is only the amorphous polymer in the cell and the cellular molecular weight is greater than the threshold strength molecular weight $M_{n c}^{\sigma}$, the strength phase of this cell is called the amorphous strength phase. The threshold strength molecular weight is discussed below. The strength change in the amorphous phase is most affected by molecular weight. A scaling relation between the molecular weight and the tensile strength can be used here and the relation is denoted as following:

$$
\sigma_{\mathrm{A}}(i, j, t)=\sigma_{\mathrm{A} 0} \times \alpha_{1}\left(\frac{M_{n \mathrm{~A}}(i, j, t)}{M_{n 0}}\right)^{\beta_{1}}
$$

Here $\sigma_{\mathrm{A}}(i, j, t)$ stands for the strength of amorphous cell $(i, j)$ at time $t, \sigma_{\mathrm{A} 0}$ stands for the initial strength, $M_{n \mathrm{~A}}(i, j, t)$ is the molecular weight of amorphous strength phase cell( $(i, j)$ at time $t$, $M_{n 0}$ is the initial molecular weight, $\alpha_{1}$ and $\beta_{1}$ are constants related to the material itself.

2) The strength vacancy phase

When cavities appear due to oligomers diffusing, the cells are holes, and there is no strength in these cells of holes. Furthermore, it is believed that when the molecular weight of an amorphous polymer falls to a threshold $M_{n c}^{\sigma}$, the cell will no longer contribute to the poly- 


\section{ACCEPTED MANUSCRIPT}

mer strength, i.e. the value of the cellular strength equals zero.

$$
\sigma(i, j, t)=0 \quad \text { when } M_{n}(i, j, t) \leq M_{n C}^{\sigma}
$$

The threshold molecular weight $M_{n \mathrm{c}}^{\sigma}$ is relative with material properties.

3) The crystalline strength phase

The formation of crystals increases the strength of polymers. The strength of the crystallised cell is marked as $\sigma_{c}(i, j, t)$. The value of $\sigma_{c}(i, j, t)$ will be relevant to the molecular weight. A scaling law is also used here to denote the strength of the crystallised phase.

$$
\sigma_{\mathrm{c}}(i, j, t)=\sigma_{\mathrm{co}} \times \alpha_{2}\left(\frac{M_{n c}(t)}{M_{n 0}}\right)^{\beta_{2}}
$$

Here $\sigma_{\mathrm{C}}(i, j, t)$ stands for the strength of a crystalized cell $(i, j)$ at time $t, \sigma_{\mathrm{co}}$ stands for the initial strength, $M_{n c}(i, j, t)$ is the molecular weight of crystalized cell $(i, j)$ at time $t, M_{n 0}$ is the initial molecular weight, $\alpha_{2}$ and $\beta_{2}$ are constants related to the material itself.

The three phases play different roles in supporting the strength during the degradation, and the strength of cell $(i, j)$ at time $t$ can be expressed as $\sigma(i, j, t)$ :

$$
\sigma(i, j, t)= \begin{cases}\sigma_{\mathrm{A}}(i, j, t) & \text { the amorphous strength phase } \\ 0 & \text { the non-strength phase } \\ \sigma_{C}(i, j, t) & \text { the crystalline strength phase }\end{cases}
$$

\subsection{Coupling of Multiscale Heterogeneous Strength Model}

The macro polymer device is discretized as $n \times n$ mesoscopic scope cellular grids. The macro physical properties (e.g. molecular weight, strength) can be obtained from the statistics of all cells' properties. After the computation of micro chain scission and recrystallization, mesoscopic cellular statuses are updated, cellular strength phases are updated, and the number of chains in each cell is recorded too. The physical property of number average molecular weight, the number of chains, and strength of cells are recorded as $M_{n}(i, j, t), N(i, j, t)$ and $\sigma(i, j, t)$ respectively for cell $(i, j)$ at time $t$. From statistics of all mesoscopic cells $n \times n$, the average molecular weight $\overline{M_{n}}$, crystallinity degree $X_{c}(t)$ and the macro normalized device strength $\bar{\sigma}(t)$ can be obtained. The device strength is computed from the statistics of all cells' strength:

$$
\bar{\sigma}(t)=\frac{\sum_{i=1}^{n} \sum_{j=1}^{n} \sigma(i, j, t) / \sigma_{0}}{n \times n} \quad(i, j=1,2, \ldots, n)
$$

For cells with different heterogeneous phases, Eq. (10) is replaced by the following:

$$
\bar{\sigma}(t)=\frac{\sum \sigma_{\mathrm{A}} / \sigma_{0}}{n \times n}+0+\frac{\sum \sigma_{\mathrm{C}} / \sigma_{0}}{n \times n}
$$

The first item in the right side of Eq.(11) is the role of the amorphous strength phase, the second item is the vacancy phase that has no strength, and the third item is the role of the crystalline strength phase. Combining Eq. (6) and Eq. (8), Eq. (11) can also be rewritten as:

$$
\bar{\sigma}(t)=X_{\mathrm{A}}(t) \alpha_{1}\left(\frac{M_{n \mathrm{~A}}(t)}{M_{n 0}}\right)^{\beta_{1}}+X_{\mathrm{c}}(t) \alpha_{2}\left(\frac{M_{n \mathrm{C}}(t)}{M_{n 0}}\right)^{\beta_{2}}
$$




\section{ACCEPTED MANUSCRIPT}

Here $X_{\mathrm{A}}(t)$ is computed by statistics of amorphous strength phases zones which is comprised by cells with amorphous status $(x(i, j, t)=1)$ or chain scission status $(x(i, j, t)=0)$ with molecular weight more than threshold strength molecular weight.

$$
X_{\mathrm{A}}(t)=\frac{\sum_{i=1}^{n} \sum_{j=1}^{n} \varphi(i, j, t)}{n \times n} \quad \varphi\left(i, j, t \neq \begin{cases}1 & \text { the amorphous stre } 1 \\ 0 & \text { not amorphous stren! }\end{cases}\right.
$$

And $X_{c}(t)$ is the same statistics computation of crystallised cells. Therefore taking the average of all the cells strength values, the overall macro device strength of polymer matrix will be obtained.

With the accumulation of oligomers, these oligomers can diffuse out of the device. The diffusion is modelled by using Fick's laws at the macroscopic scale $[16,21,23]$.

$$
\frac{d c_{\mathrm{o} 1}}{d t}=\frac{d R}{d t}+\operatorname{div}\left[D \operatorname{grad}\left(c_{\mathrm{ol}}\right)\right]
$$

Here $c_{\mathrm{ol}}$ is the oligomers concentration. $R_{\mathrm{ol}}(t)$ is the oligomers counted by all the cells. $D$ is the diffusion coefficient which is influenced significantly by the pores produced by degradation [21].

$$
D=D_{\mathrm{P}}+\left(1.3 \varepsilon^{2}-0.3 \varepsilon^{3}\right) \times\left(D_{\varepsilon}-D_{\mathrm{P}}\right)
$$

where $D_{\mathrm{P}}$ is the diffusion coefficient in polymer matrix, $\varepsilon$ is porosity, $D_{\varepsilon}$ is the diffusion coefficient in pores which is estimated by calculation[21]. In a polymer matrix, there may coexist amorphous and crystallised phases. The diffusion coefficient in the crystalline phase is minimal and taken as zero. So

$$
D_{\mathrm{P}}=D_{0}\left(1-X_{\mathrm{C}}\right)
$$

$D_{0}$ is the oligomers diffusion coefficient in polymer amorphous domain.

The porosity $\varepsilon$ can be inferred by cellular status (hole status). The statistics of all holes number is $Z(t)$ :

$$
Z(t)=\sum_{i=1}^{n} \sum_{j=1}^{n} \psi(i, j, t) \quad \psi\left(i, j, \neq \begin{cases}1 & x(i, j, t)=-1 \\ 0 & x(i, j, t) \neq-1\end{cases}\right.
$$

then $\varepsilon(t)=\frac{Z(t)}{n \times n}$

Oligomers diffusion plays a considerable role in the mass loss of the material. Another part of the overall weight loss is the small molecules solution. The critical degree of small molecules $\vartheta$ may be relative to polymer material properties, temperature and pressure. There is no precise formula to get the value $\vartheta$ of different materials in different solvent so far. Here from the calculation of fitting the experiment data, the critical degree of small molecules $\vartheta$ is set for different fitting cases that are shown in Table 1.

After oligomer diffusion and small molecular solution, the remaining number of oligomers in 


\section{ACCEPTED MANUSCRIPT}

the polymer device is recorded as $R_{\mathrm{ol}}(t+1)$. The chains number and the molecular weight are both updated to $N(i, j, t+1)$ and $M_{n}(i, j, t+1)$. This will be the new starting values for the next iteration. In summary, the multi-scale heterogeneous strength model can simulate the discrete mesoscopic cellular strength phases evolution, cellular status evolution, the microscopic chains scission reaction, autocatalytic reaction, recrystallization, and the macroscopic oligomers diffusion of polyester degradation. The flow chart of the asynchronous modeling method is shown in Fig. 1. And the computer program can be executed as the following steps:

Step 1: Initialization: set $n$ and the initial cellular statuses, the initial molecular weight, degree of crystallization and other polymer characteristics (the input of program);

Step 2: Set the chain scission iteration time $t_{s}=0$;

Step 3: Determine the location and the time of the chain cleavage by the Monte Carlo method and cellular statuses of neighbors, and then execute the chain scission;

Step 4: Execute the chain crystallization; Update the cellular statuses;

Step 5: Judge whether the chain scission time $t_{s}$ is less than diffusion time step length $\Delta t_{d}$, if yes, go to step 6, if no, go to step 2;

Step 6: Make statistics for all cellular oligomers $R_{\mathrm{ol}}$, crystallization $X_{c}$, porosity $\varepsilon$;

Step7: Make statistics for the overall tensile strength of the device;

Step8: Execute oligomers diffusion;

Step9: Update every cell's molecular weight and the number of chains in it;

Step10: Judge whether program executing time is finished. If yes, program ends, if no, go to step2.

\section{Calculation}

In this section, data in three experiments are used to compare with the predictions in the multiscale strength model in order to verify the model. Polymer properties from the three experiments will be used as the input parameters in the strength model which include initial strength, initial number average molecular weight, and the initial crystallinity. All parameters can be found in table 1 . The computing execution steps of the modelling program are expressed in Section 2.3

\subsection{Calculate the Modeling Method with Data Obtained by Tsuji [24]}

In the experiment of degradation of PLLA film carried out by Tsuji [24], the initial number average molecular weight $M_{n o}$ equals $8.667 \times 10^{4} \mathrm{~g} / \mathrm{mol}$, and the initial degree of crystallinity $X_{c 0}$ is $46 \%$. The model parameters used for this computation are listed in Case 1 of Table 1 . Among all parameters, $\pi_{v 1}$ and $\pi_{v 2}$ are the parameters controlling degradation rate, $D_{0}, D_{\varepsilon}$ are the parameters controlling diffusion rate. They are related to the material properties. Here, they are obtained by referring the values of Ref. [23] and parameters optimization. It is generally believed that short chains with less than 8 degree of polymerization become water soluble and mobile [25], therefore we choose $m=8$ in all the cases here. $M_{n c}$ is the threshold molecular weight of porous appearance (the cellular status changes from 0 to -1 ), here the value of $M_{n c}$ was obtained by estimating the extending line of experimental strength with molecular weight [24]. The 
threshold degree of small molecules solution $\vartheta$ is obtained from best fitting the weight loss to the experimental data, in this case $\vartheta$ is set as $3 . M_{n c}^{\sigma}$ is estimated from the fitting result with the experimental data. The parameters $\alpha_{1}$ and $\beta_{1}, \alpha_{2}$ and $\beta_{2}$ in Eq.(12) are relevant to the material properties. The cellular scale $n$ is set to 1000 . The model parameters used for this analysis calculation are listed in case 1 of Table 1 . The diffusion time step is 1000s, the total time cost is less than 20 hours in Intel(R) Core(TM) i7-4790 CPU, 20.0GB memory computer.

The strength phase evolution is shown in Fig.2. For cells $1000 \times 1000$, it is difficult to see the detailed evolution. We enlarged the area within the rectangle (shown in Figure 2) and used different colours to represent the different strength phase of the cells. Yellow represents the amorphous strength phase. Red represents the crystalline strength phase. Black represents strength vacancy phase with hole cellular status $(x(i, j, t)=-1)$. Grey represents another strength vacancy phase, in which the cellular status is the amorphous status $(x(i, j, t)=1$ or $x(i, j, t)=0)$ but its molecular weight is smaller than $M_{n c}^{\sigma}$. From the strength evolution figures, it can be seen distinctly that the strength zones change with the degradation time.

The cellular status evolution during degradation is shown in Figure 3. Green represents the initial amorphous polymer status $(x(i, j, 0)=1)$. Blue represents the cells with chain scission reaction $(x(i, j, t)=0)$. Black represents the cells with holes in them $(x(i, j, t)=-1)$. Pink represents the cells with crystallization status $(x(i, j, t)=-2)$.

The change of normalised number average molecular weight, the degree of crystallinity, the tensile strength of the film calculated by our multiphase model and the experimental data are shown in Fig.4. From the comparison in Fig.4, it can be seen that the modelling results fit with the experiment result very well.

\subsection{Calculate the Modelling Method with Data Obtained by Tsuji and Muramatsu [26]}

In this case, the experiment by Tsuji and Muramatsu [26]( PLLA (60\%) and poly(vinyl alcohol) (PVA 40\%) is chosen to analysis the validity of the multiphase strength model for copolymers. The material properties are $M_{O P L A A}=72 \mathrm{~g} / \mathrm{mol}, M_{O P V A}=44 \mathrm{~g} / \mathrm{mol}$, the initial number average molecular weight $M_{n}=9.26 \times 10^{4} \mathrm{~g} / \mathrm{mol}$. The model parameters used for this analysis calculation are listed in case 2 of Table 1 . The initial degree of crystallization is 0 , and due to the long computation time we set $n=700$ in this case. The threshold molecular weight $M_{n c}$ was obtained by estimating the extending line of experimental strength with molecular weight [23]. The degradation rate parameters $\pi_{v 1}$ and $\pi_{v 2}$, diffusion rate parameters $D_{0}, D_{\varepsilon}$ are obtained by referring the values of Ref. [23] and parameters optimization. The critical degree of small molecules $\vartheta$ is set as 4 from best fitting the weight loss to the experimental data. The parameters $\alpha_{1}$ and $\beta_{1}$ are set as 1.23 and $1.21, \alpha_{2}$ and $\beta_{2}$ are 2.214 and 1.41 respectively from best fitting the strength change to the experimental data.

With the degradation process, the cellular strength phase evolution is shown in Fig.5. The fitting between the heterogeneous model and the experimental data for molecular weight, the degree of crystallization and strength are shown in Fig. 6. From Fig.6, the calculated values of the multi-scale heterogeneous model align with the experiment result very well.

\subsection{Testing the Modelling Method Using Data Obtained by Duek et al. [27]}

To validate the heterogeneous multi-scale strength model and evaluate the main strength 


\section{ACCEPTED MANUSCRIPT}

parameters $\alpha_{1}, \beta_{1}, \alpha_{2}$ and $\beta_{2}$, the experiments of two samples by Duek and coworkers [27] are chosen. The chosen samples in experiments are amorphous poly(lactic acid) pin (case 3(a)) and semi-crystalline poly(lactic acid) pin (case 3(b)). In case 3 (a) and 3 (b), $M_{P L A}$ equals $72 \mathrm{~g} / \mathrm{mol}$, and the initial number average molecular weights $M_{n o}$ are $1.52 \times 10^{5} \mathrm{~g} / \mathrm{mol}$ and $1.53 \times 10^{5} \mathrm{~g} / \mathrm{mol}$ respectively. The initial strength are 190.4 MPa and 195.0 MPa respectively listed in Table 1. The model parameters used for this analysis calculation are listed in case 3 of Table $1 . n$ is set to 1000 in this case. The parameters $\alpha_{1}$ and $\beta_{1}, \alpha_{2}$ and $\beta_{2}$ in Eq.(12) are relevant to the material properties, in this case they are selected to be the same.

In the first sample (case $3(\mathrm{a})$ ), the value of $\alpha_{1}$ and $\beta_{1}$ are determined to fit the experiment data. The values are 1.35 and 3.6 respectively. The evolution result of cellular strength phase of degradation is shown in Fig.7. From the strength evolution figures, it can clearly be seen that the strength zones change with the degradation time. The cellular status evolution of degradation in case 3(a) is shown in Figure 8. The numerical values of molecular weight, the degree of crystallinity, the changes of tensile strength with the degradation time calculated by the multiscale multiphase strength model and the experiment result are plotted in Fig.9. From the comparison of Fig.9, the calculation values of the model align with the experimental results very well.

With all the model parameters of case 3(a) unchanged for the second sample (case 3(b)) degradation computation (except the recrystallization probability $p$ ). The cellular strength phase evolution of degradation in case $3(\mathrm{~b})$ is shown in Fig.10. From the strength evolution figures, it can be seen that different strength zones change differently during the degradation process. The cellular status evolution of degradation in case $3(b)$ is shown in Figure 11. The numerical values of molecular weight, degree of crystallinity, the changes of strength with the degradation time are calculated by the multiphase strength model and the experiment result are plotted in Fig.12. From the comparison of Fig.12, the calculation values of the model align with the experiment results very well.

From the degradation comparison of PLA without initial crystalline (case 3(a)) and with initial crystalline (case 3(b)), the multiscale heterogeneous strength model captures the effect of different strength zones. The strength of PLA with initial crystalline (case 3(b)) dissipate faster than PLA without initial crystalline, which can be explained by autocatalytic reaction (oligomers are accumulated inside by the crystalline cells). The model calculation result fit the experimental results very well. Our heterogeneous strength model can simulate the change of polymer tensile strength, molecular weight, the degree of crystallinity during the hydrolysis reaction.

\section{Discussion}

In case 3(a) mentioned in section 3.3, the device has almost no crystals at the beginning [27], but at week 4 of degradation, the degree of crystallinity increases sharply to $42 \%$. That means during this period, the number of the polymer cells with crystalline status $((x(i, j, t)=-2)$ significantly rises. According to our strength model, the crystallised cells have higher strength compared to amorphous cells. Therefore, after 4 weeks, the overall strength of the polymer device increases even though the overall molecular weight reduces. And when the degradation reaction proceeds to the later stage, more and more cells have a molecular weight lower than the critical threshold. Meanwhile, the experiment data shows the strength at this stage will drop to a very low level. Therefore, the many newly-formed cells with the strength vacancy phases may be the 


\section{ACCEPTED MANUSCRIPT}

reason why the strength drops significantly at the later stage. It can be seen from figure 9 that the heterogeneous strength model can capture the main degradation mechanisms for amorphous PLA samples.

The same set of model parameters used in case 3(a) is applied to case 3(b) except $p$, the recrystallization probability. In case $3(b)$, the initial crystallization of PLA is $48 \%$. The large amount of crystals reduces the mobility of polymer chains. Thus the recrystallization probability $p$ was reduced from 0.05 in case 3(a) to 0.01 in case 3(b). Degradation rate of samples in case 3(b) is higher than that of samples in case 3(a) during the first two weeks. From the model, we can understand that the initial crystallinity reduces the diffusion coefficient of oligomers calculated in equation (16), resulting in higher concentration of oligomers with carboxylic acid end groups in the device, thus higher degradation rate at the beginning of degradation. Fast degradation in case 3(b) leaves more cavities in the device than slow degradation in case 3(a) as shown in figure 10. The cavities contribute to a greater diffusion coefficient according to equation (15) of oligomers after week two, thus a slower degradation rate in case 3(b) than in case 3(a). As a result of the initial crystals, the strength of samples in case $3(\mathrm{~b})$ is higher than that in case $3(\mathrm{a})$. In contrast to case 3(a), no strength increasing was detected either by experiments or by predictions from the model after degradation. This is due to fast degradation during the first two weeks. Therefore, the multiscale heterogeneous strength model can provide more details to understand the strength change mechanism during degradation.

As for the case mentioned in section 3.1, PLLA with a high degree of initial crystallinity was used [24]. In the whole process of its degradation, the upward trend of its degree of crystallinity is relatively gentle. The contribution of crystallization to strength is always smaller than the effect of molecular weight's reduction on strength in this case, so the normalized tensile strength has been declining throughout the whole degradation process.

In section 3.2, polymer with 60\% PLLA and 40\% PGA was used [26] to prove that the heterogeneous strength calculating method also applies to copolymers. The calculated values of the multiphase strength model fit with the experimental results very well.

We believe the porous, crystalline and amorphous phases play different roles in determining the mechanical strength of the polymer, and the multiphase method to predict every cellular strength by its status is reasonable.

To analyze the sensitivity of this heterogeneous strength model, the parameters $\alpha_{1}$ and $\beta_{1}$ are selected by different values (case 1 is as the computation example) and the root mean square error (rmse) for normalized strength with the change of $\alpha_{1}$ and $\beta_{1}$ are shown in Fig. 13. From Fig.13, it can be seen that $\alpha_{1}, \beta_{1}$ converges to a stable range. Moreover, we set different values for threshold molecular weight $M_{n c}^{\sigma}$ using case 2 as an example. The result of root mean square error of strength with different values of threshold molecular weight $M_{n c}^{\sigma}$ was shown in Fig. 14.

From Fig.14, it can be seen that $M_{n c}^{\sigma}=5000$ converges to a stable and acceptable error. The computation time is about 10-15 hours to execute the whole degradation, and the length of time mostly depends on the material properties and the size of cellular grid. In general, a material with larger molecular weight requires more time degrade. Meanwhile, the size of cellular grid, i.e. the value of $n$, affects computation time. If $n$ is larger, the program needs more memory for the larger 


\section{ACCEPTED MANUSCRIPT}

number of cells, and picking cells and chains to participate in the reaction by Monte Carlo method will take more time. But obviously, the larger $n$ is, the more accurate the modelling result is. So the value of $n$ should be neither too small nor too large. The parameter $n$ with the root mean square error is shown in Fig. 15. And the calculation time with different parameters $n$ is shown in Fig. 16. From the comparison of Fig.15 and Fig.16, it can be seen clearly that the chosen of parameter $n$ is based on the time cost and accuracy requirement. Case 2 is selected as the example for the sensitivity analysis about parameter $p$. The root mean square error of weight loss, normalized molecular weight, crystallinity and strength with different values of $p$ were shown in Fig. 17. It can be seen that $p=0.01$ leads to the least crystallinity error, strength error, weight loss error and normalized molecular weight error.

Polymer strength change during degradation is complicated for the dynamic change of recrystallization and porosity due to the micro chain scission. Polymer strength should obey the extreme value statistics due to the heterogeneous phases, especially strength vacancy phase. It deserves more research in this area.

\section{Conclusions}

A multiscale heterogeneous strength model is presented in this paper to demonstrate the different strength phase roles that contribute to the tensile strength during polymer bulk erosion degradation. The heterogeneous strength model is coupled with micro chain scission, recrystallization caused by chain scission, mesoscopic cellular status and macro oligomers diffusion equation by integrated continuous, discrete, stochastic, definite modeling methods. The strength phase evolution and distribution with time, the evolution of polymer inner shape, molecular weight and its distribution with time, chain number with time, the degree of crystallinity with time can be simulated simultaneously from this multiscale heterogeneous strength model. Several experimental data were used to validate the model. As contrast, PLA with and without initial crystals were used. All model parameters were obtained by fitting curves with the experimental data in case 3(a). These model parameters are then applied to case 3(b). The calculation results fit the experiment data of case $3(b)$ very well. This result shows that the model is validated and can predict strength change during degradation. Additionally, the model gives more details to understand the mesostructure change during degradation. Together with case 1 and case 2 , it can conclude that the model can be used to predict strength change during degradation for amorphous polymers, semi-crystalline polymers and copolymers.

\section{Acknowledgement}

This paper is sponsored by the National Study Abroad Fund of China and supported by The National Key Research and Development Program of China (2017YFB1002304). 


\section{Statement of Significance}

The original work in this paper is a multi-scale method (including micro scale, mesoscopic scale, macro scale and their coupling) for modeling heterogeneous strength change during degradation of bioresorbable polyesters and provides understanding to the strength change during degradation of biodegradable polymers. The result denotes the solution is reliable.

As we know, there have no papers recently to implement strength study during degradation in three scales modeling and its coupling.

From our model, the strength distribution during degradation, strength phrase evolution, molecular weight distribution, chain number, degree of crystallinity, the evolution of polymer inner shape, weight loss can be obtained from the calculation of the three scale heterogeneous strength model.

\section{References}

[1] R. J. Mondschein, A. Kanitkar, C. B. Williams, S. S. Verbridge, T. E. Long, Polymer structure-property requirements for stereolithographic 3D printing of soft tissue engineering scaffolds, Biomaterials, 140 (2017) 170-188.

[2] S. Stratton, N. B. Shelke, K. Hoshino, S. Rudraiah, S. G. Kumbar, Nanoscale hydroxyapatite particles for bone tissue engineering, Acta Biomater. 7(7)(2011) 1769-2781.

[3] J. C. Middleton, A. J. Tipton, Synthetic biodegradable polymers as orthopedic devices, Biomataterials 21(23)(2000) 2335-2346.

[4] T. V. Chirila, P. E. Rakoczy, K. L. Garrett, X. Lou, I. J. Constable, The use of synthetic polymers for delivery of therapeutic antisense oligodeoxynucleotides, Biomaterials, 23(2) (2002) 321-342.

[5] A. C. Vieira, R. M. Guedes, A. T. Marques, Development of ligament tissue biodegradable devices: a review, J. Biomech. 42(2009) 2421-2430.

[6] H. Tsuji, In vitro hydrolysis of blends from enantiomeric poly(lactide)s. Part 4: well-homocrystallized blend and nonblended flms, Biomaterials, 24(2003) 537-547.

[7] Vieira A C, Vieira J C, Ferra J M, et al. Mechanical study of PLA-PCL fibers during in vitro degradation[J]. Journal of the mechanical behavior of biomedical materials, 4 (2011) 451-460.

[8] Paakinaho K, Heino H, Väisänen J, et al. Effects of lactide monomer on the hydrolytic degrada- 


\section{ACCEPTED MANUSCRIPT}

tion of poly (lactide-co-glycolide) 85L/15G[J]. Journal of the mechanical behavior of biomedical materials, 2011, 4(7): 1283-1290.

[9] H. Tsuji, Y. Ikada, Properties and morphology of poly(L-lactide) 4. Effects of structural parame ters on long-term hydrolysis of poly(L-lactide) in phosphate-buffered solution, Polym. Degrad. Stab. 67(2000) 179-189.

[10] Grizzi I, Garreau H, Li S, et al. Hydrolytic degradation of devices based on poly (DL-lactic acid) size-dependence[J]. Biomaterials, 16(1995) 305-311.

[11] A. Gleadall, J. Pan, H. Atkinson, A simplified theory of crystallization induced by polymer chain scissions for biodegradable polyesters, Polym. Degrad. Stab. 97 (2012) 1616-1620.

[12] Y. Wang, X. Han, J. Pan, An entropy spring model for the Young's modulus change of biode gradable polymers during biodegradation, J. Mech. Behav. Biomed. Mater. 3(1) (2010) 14-21.

[13] A. Gleadall, J.Pan, M. A. Kruft. An atomic finite element model for biodegradable polymers. Part 2. A model for change in Young's modulus due to polymer chain scission. J. Mech. Behav. Biomed. Mater. 51 (2015) 237-247.

[14] H. Samami, J. Pan, A constitutive law for degrading bioresorbable polymers, J. Mech. Behav. Biomed. Mater. 59 (2016) 430-445.

[15] R.N. Shirazi, W. Ronan, Y. Rochev, P. McHugh, Modelling the degradation and elastic proper ties of poly(lactic-co-glycolic acid) films and regular open-cell tissue engineering scaffolds, J. Mech. Behav. Biomed. Mater. 54 (2016) 48-59.

[16] Y. Wang, J. Pan, X. Han, C. Sinka, L. Ding, A phenomenological model for the degradation of biodegradable polymers, Biomataterials 29 (2008) 3393-3401.

[17] Q. Breche, G. Chagnon, G. Machado, B. Nottelet, X. Garric, E. Girard, D. Favier, A non-linear viscoelastic model to describe the mechanical behavior's evolution of biodegradable polymers during hydrolytic degradation, Polym. Degrad. Stab. 131 (2016) 145-156.

[18] Q. Breche, G. Chagnon, G. Machado, E. Girard, B. Nottelet, X. Garric, D. Favier, Mechanical behaviour's evolution of a PLA-b-PEG-b-PLA triblock copolymer during hydrolytic degradation, J. Mech. Behav. Biomed. Mater. 60(2016) 288-300

[19] P.J. Folry, Tensile strength in relation to molecular weight of high polymers, Journal of the American Chemical Society, 67(1945) 2048-2050.

[20] D. F. Farrar, R. K. Gillson, Hydrolytic degradation of polyglyconate B: the relationship between degradation time, strength and molecular weight, Biomataterials 23(16) (2002) 3905-3912.

[21] J. Pan, Modelling Degradation of Bioresorbable Polymeric Medical Devices, Woodhead Pub lishing Ltd., Cambridge, UK, 2014.

[22] S. K. Saha, H. Tsuji, Effects of rapid crystallization on hydrolytic degradation and mechanical properties of poly(L-lactide-co-e-caprolactone), React. Funct. Polym. 66 (2006) 1362-1372.

[23] T. Zhang, S. Zhou, X. Gao, Z. Yang, L. Sun, D. Zhang, A multi-scale method for modeling deg radation of bioresorbable polyesters, Acta Biomater. 50 (2017) 462-475.

[24] H. Tsuji, In vitro hydrolysis of blends from enantiomeric poly(lactide)s Part 1. Well-stereo-complexed blend and non-blended films, Polymer 41 (2000) 3621-3630.

[25] Buchanan F. Degradation rate of bioresorbable materials: prediction and evaluation. Cambridge, England/Boca Raton [FL]: Woodhead Pub./CRC Press; 2008.

[26] H. Tsuji, H. Muramatsu, Blends of aliphatic polyesters: $V$ Non-enzymatic and enzymatic hy drolysis of blends from hydrophobic poly(I-lactide) and hydrophilic poly(vinyl alcohol), Polym. 


\section{ACCEPTED MANUSCRIPT}

Degrad. Stab. 71 (2001) 403-413.

[27] E.A.R. Duek, C.A.C. Zavaglia, W.D. Belangero, In vitro study of poly(lactic acid) pin degrada tion, Polymer 40 (1999) 6465-6473.

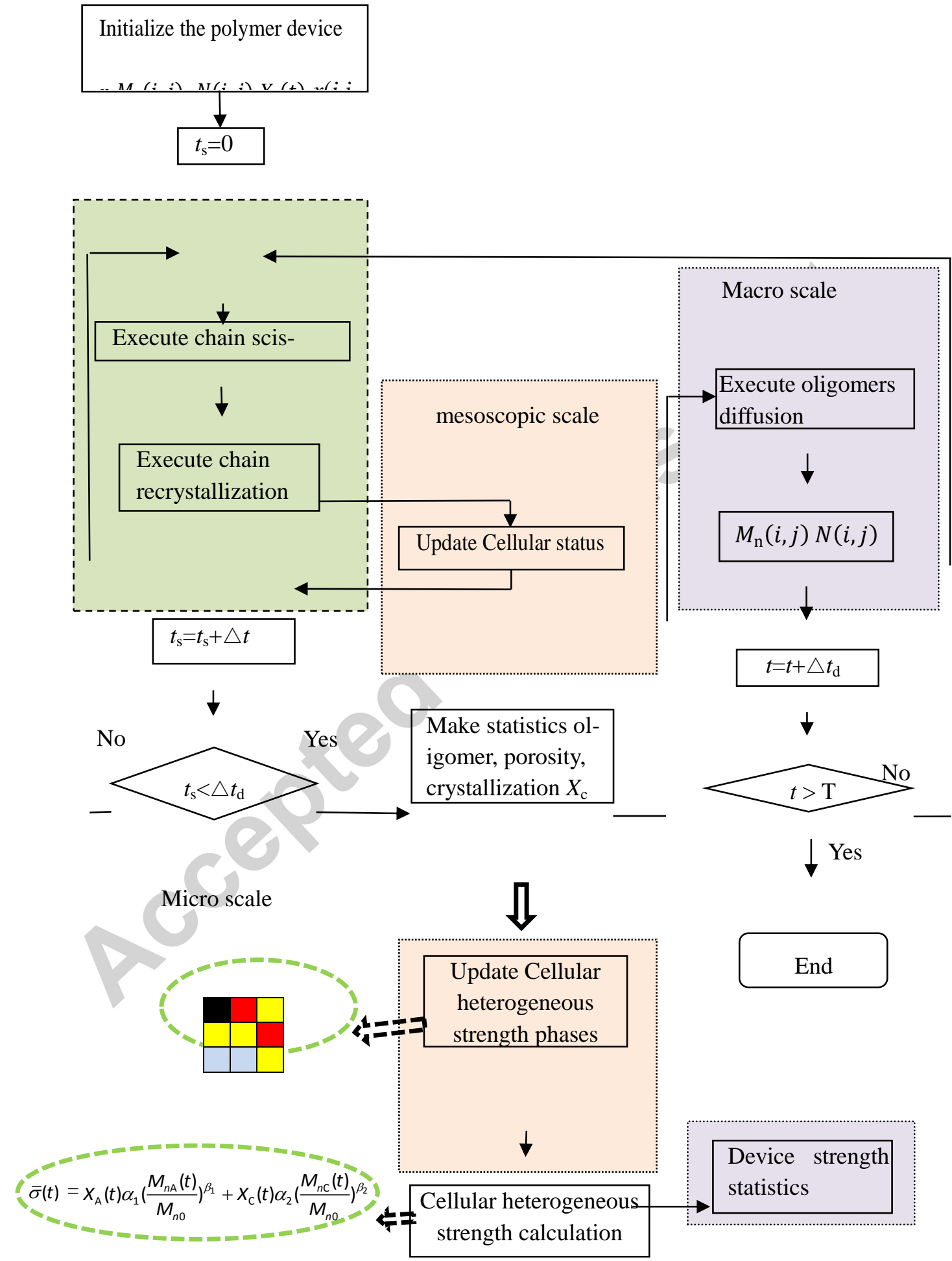




\section{ACCEPTED MANUSCRIPT}

Fig.1. the computation multiscale heterogeneous strength model and the coupling for polymer degradation

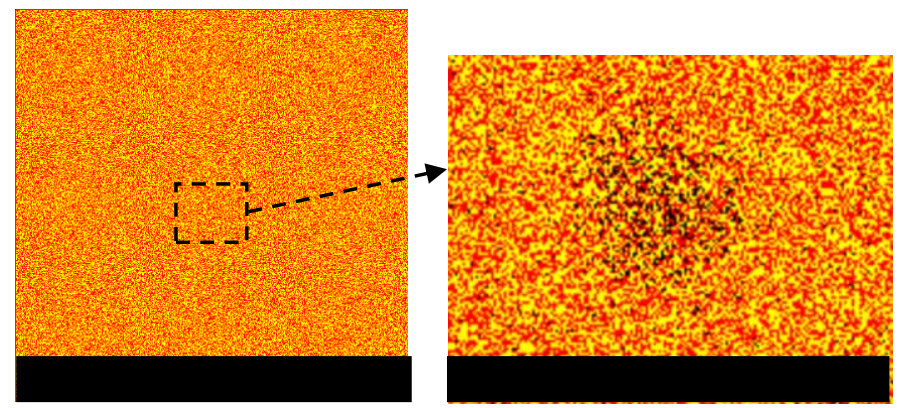

0 month

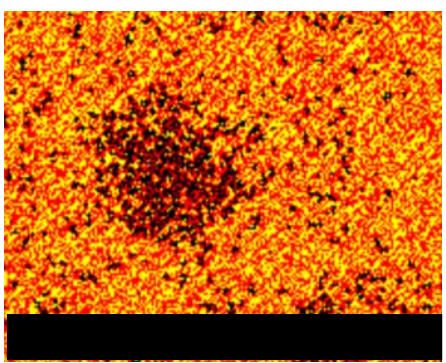

20 months
4 months

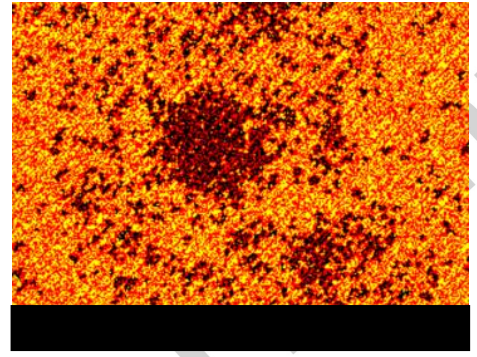

24 months

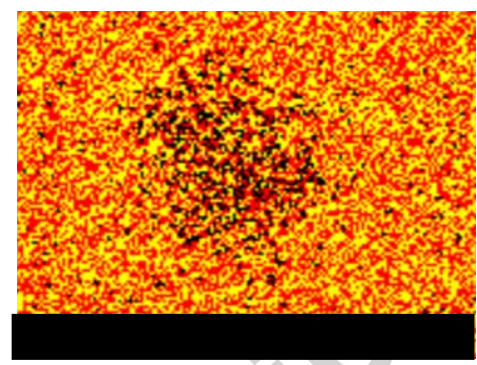

12 months

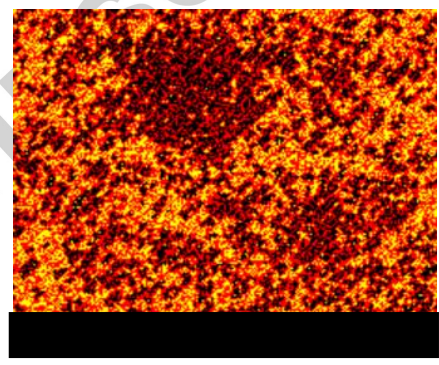

30 months

Fig.2. The cellular strength phase evolution of the degradation process of the polymer sample in Ref.[24]. Yellow represents the amorphous strength phase. Red represents the crystalline strength phase. Grey represents the strength vacancy phase with amorphous cellular status $(x(i, j, t)=1$ or $x$ $(i, j, t)=0)$ but its molecular weight is smaller than $M_{n c}^{\sigma}$. Black represents another kind of the strength vacancy phase with hole cell status $(x(i, j, t)=-1)$.

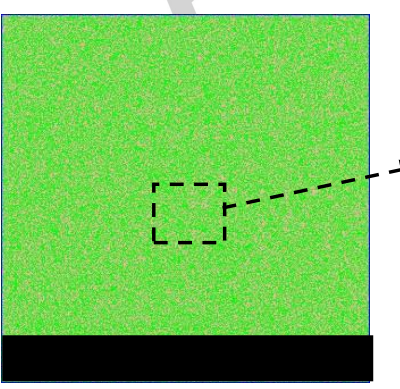

0 month

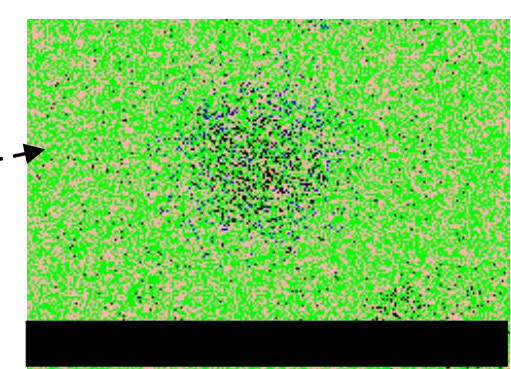

4 months

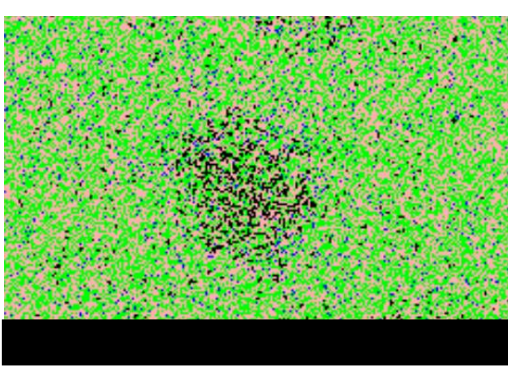

12 months 


\section{ACCEPTED MANUSCRIPT}
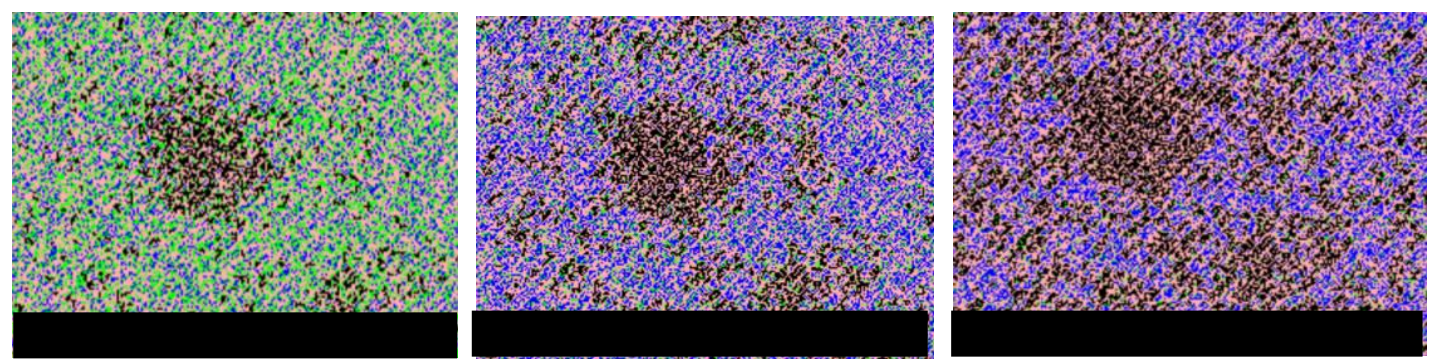

20 months

24 months

30 months

Fig.3. The cellular status evolution of the degradation process of the polymer sample in Ref.[24]. Green stands for the initial amorphous status $(x(i, j, t)=1)$. Pink stands for the cells with crystallization status $(x(i, j, t)=-2)$. Blue stands for the cells with chain scission reaction $(x(i, j, t)=0)$. Black stands for the cells with holes in them $(x(i, j, t)=-1)$.

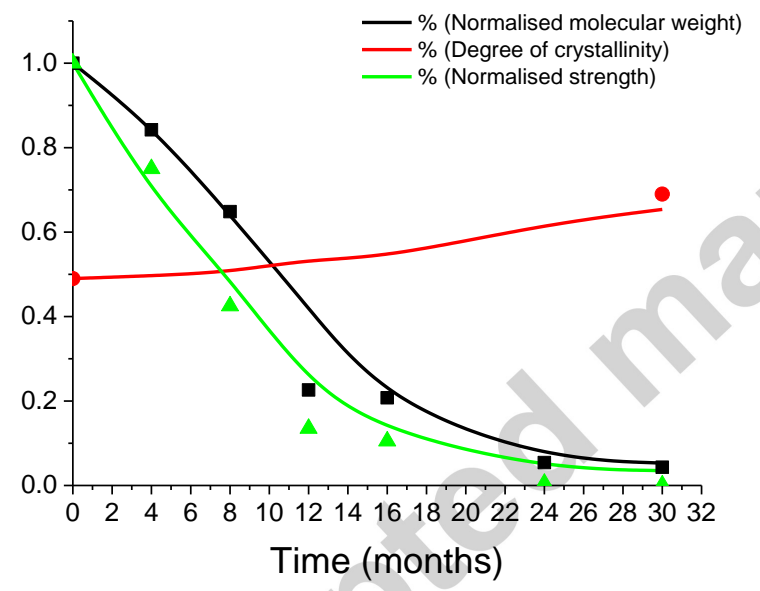

Fig.4. Comparison between the multiscale heterogeneous strength model and the experimental data obtained by Tsuji [24] for number average molecular weight, volume degree of crystallinity and strength as functions of degradation time for the polymer sample. The continuous lines represent the model prediction while the discrete symbols are the experimental data. 


\section{ACCEPTED MANUSCRIPT}

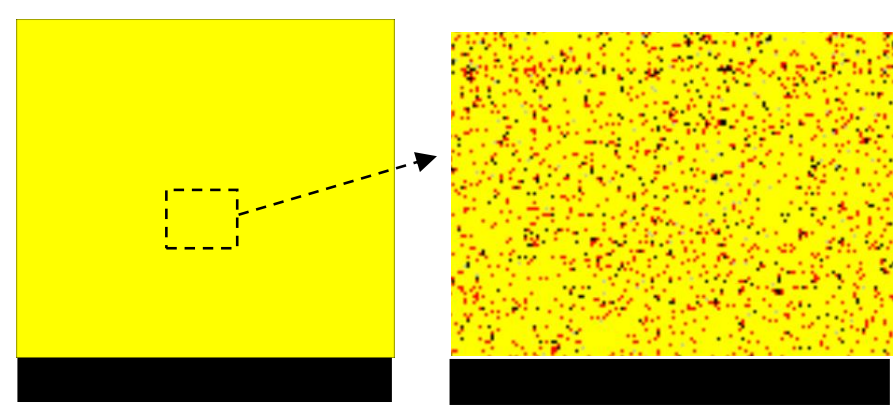

0 month

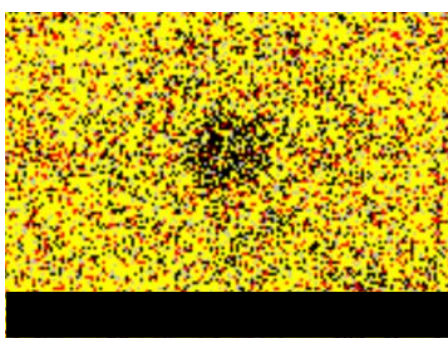

6 months
2 months

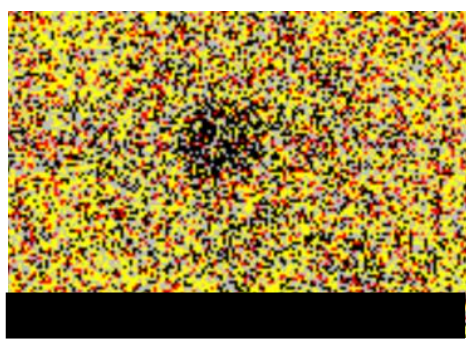

8 months

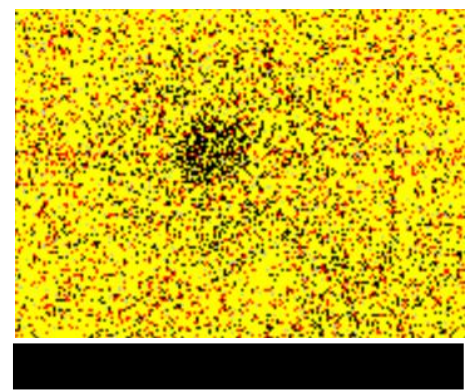

4 months

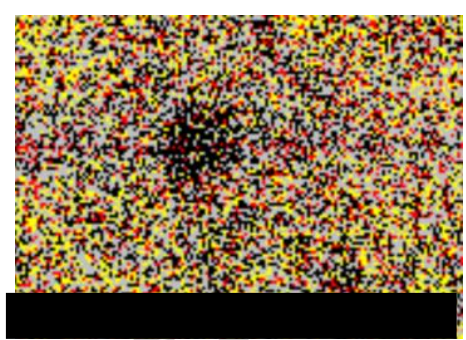

10 months

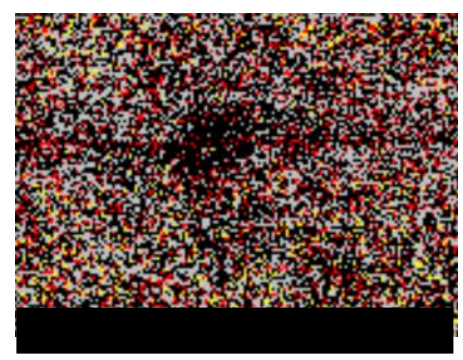

12 months

Fig.5. The cellular strength phase evolution of the degradation process of copolymer of PLLA (60\%) and PVA in Ref.[26]. Yellow represents the amorphous phase. Red represents the crystalline phase. Grey represents one kind of the strength vacancy phase which the cellular status is the amorphous status $(x(i, j, t)=1$ or $x(i, j, t)=0)$ but its molecular weight is smaller than $M_{n c}^{\sigma}$. Black represents another kind of the strength vacancy phase with hole status $(x(i, j, t)=-1)$. 


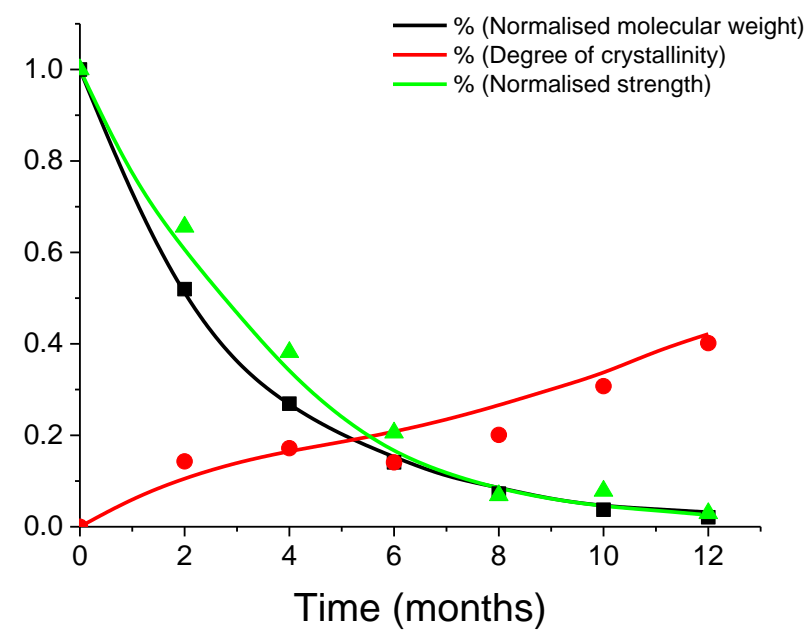

Fig.6. Comparison between the heterogeneous model and the experimental data obtained by Tsuji and Muramatsu [26] for number average molecular weight, volume degree of crystallinity and strength as functions of degradation time for the polymer sample. The continuous lines represent the model prediction while the discrete symbols are the experimental data.
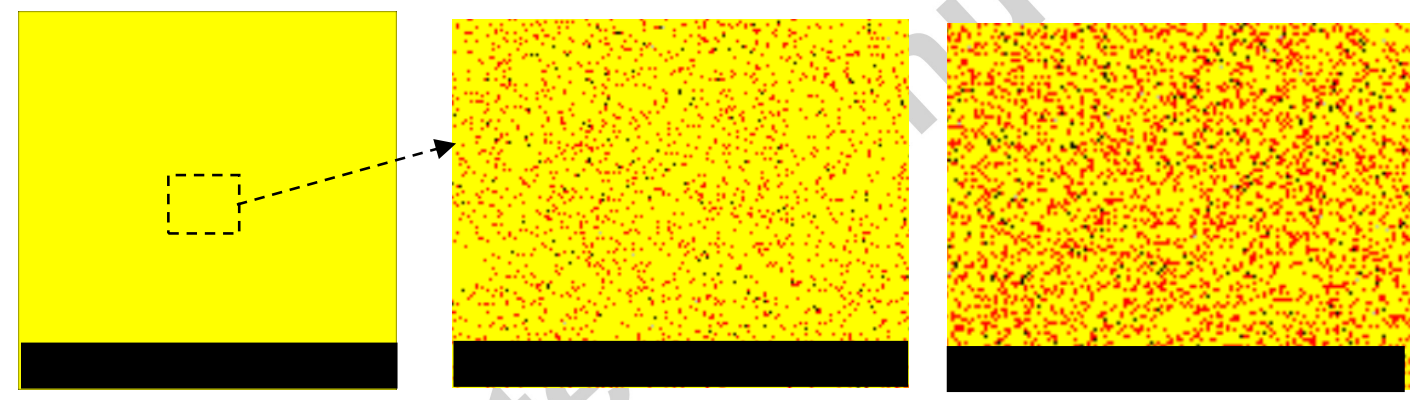

0 week

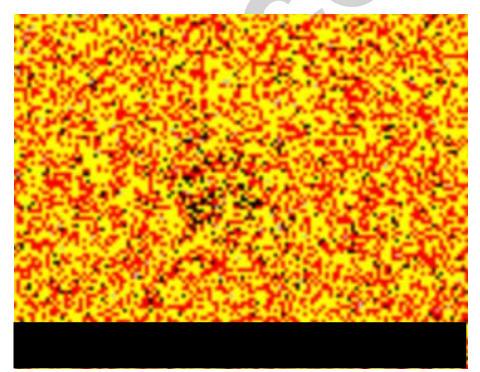

8 weeks
1 week

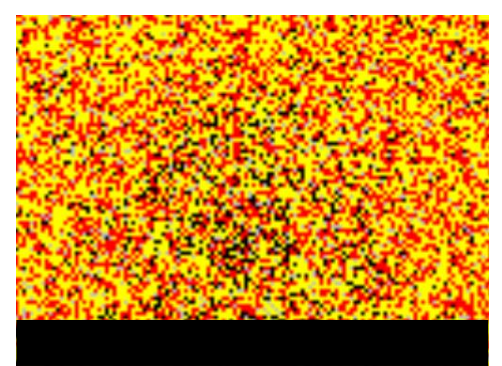

12 weeks
2 weeks

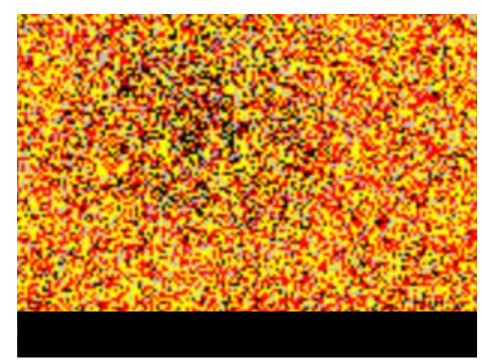

24 weeks

Fig.7. The cellular strength phase evolution of the degradation process of the PLA device without initial crystallinity in Ref.[27]( case 3(a)). Yellow represents the amorphous strength phase. Red 


\section{ACCEPTED MANUSCRIPT}

represents the crystalline strength phase. Grey represents one kind of the strength vacancy phase which the cellular status is the amorphous $\operatorname{status}(x(i, j, t)=1$ or $x(i, j, t)=0)$ but its molecular weight is smaller than $M_{n c}^{\sigma}$. Black represents another kind of the strength vacancy phase with hole status cells $(x(i, j, t)=-1)$.

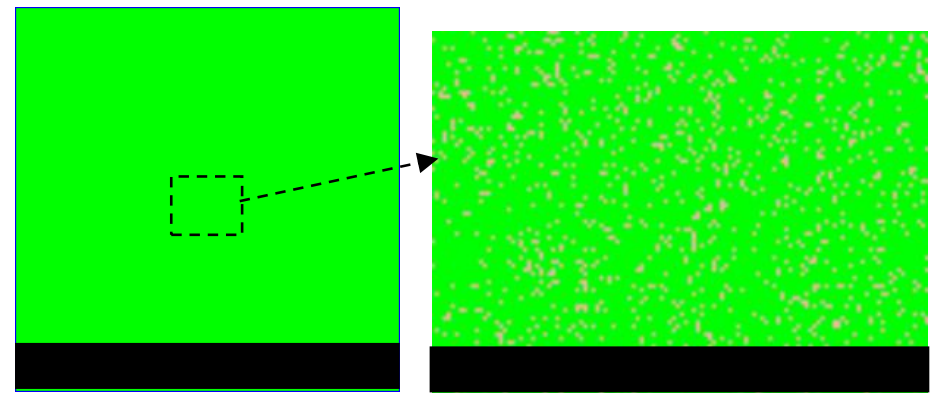

0 week

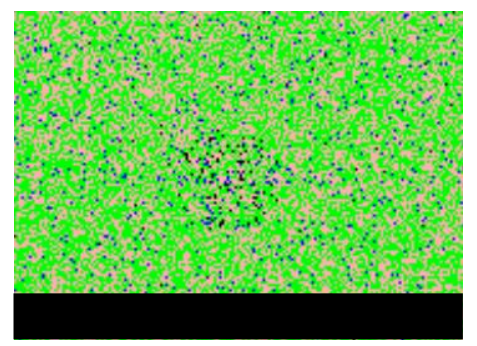

8 weeks
1 week

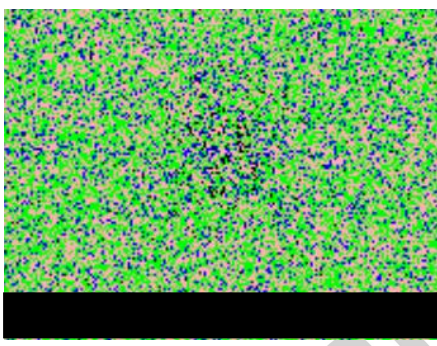

12 weeks

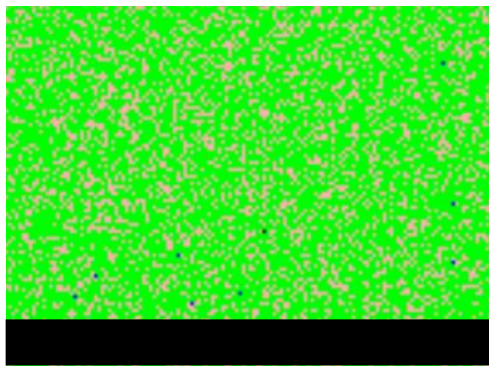

2 weeks

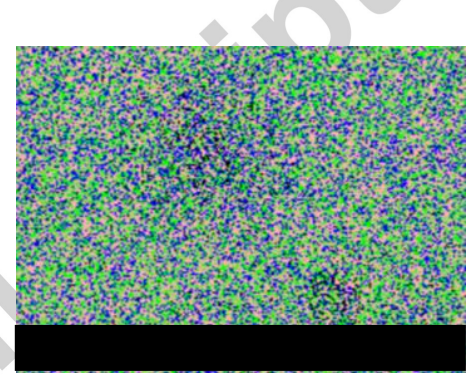

24 weeks

Fig.8. The cellular status evolution of the degradation process of PLA device without initial crystallinity in Ref.[27]( case 3(a)). Green stands for the initial amorphous status $(x(i, j, t)=1)$. Pink stands for the cells with crystallization status $(x(i, j, t)=-2)$. Blue stands for the cells with chain scission reaction $(x(i, j, t)=0)$. Black stands for the cells with holes in them $(x(i, j, t)=-1)$. 


\section{ACCEPTED MANUSCRIPT}

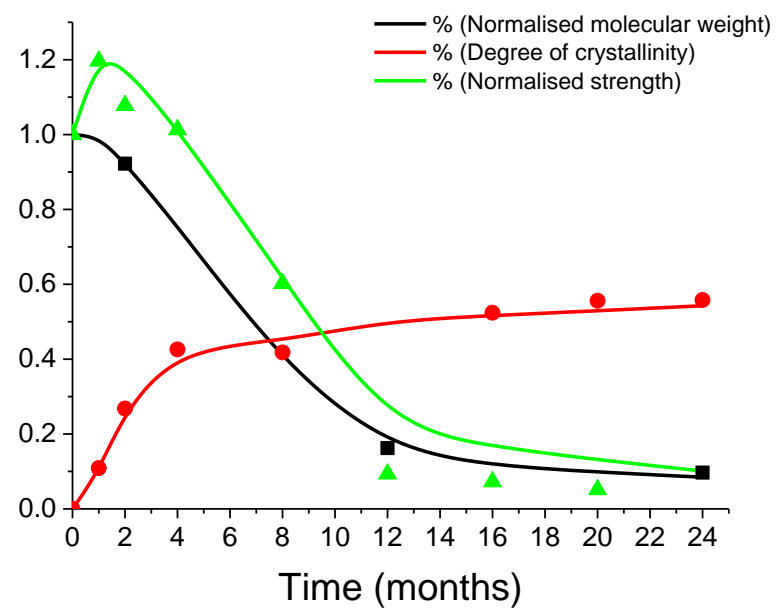

Fig.9. Comparison between the multiscale heterogeneous strength model and the experimental data of sample without initial crystallinity (case 3(a)) obtained by Duek et al. [27] for number average molecular weight, volume degree of crystallinity and strength as functions of degradation time for the polymer sample. The continuous lines represent the model prediction while the discrete symbols are the experimental data.

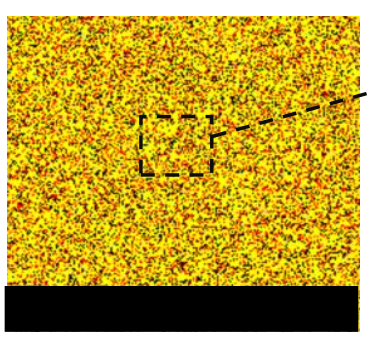

0 week

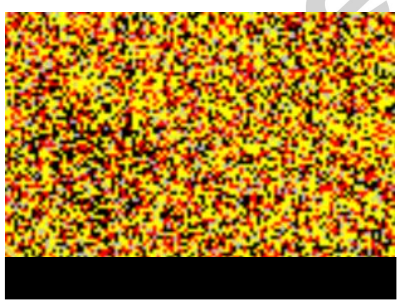

8 weeks

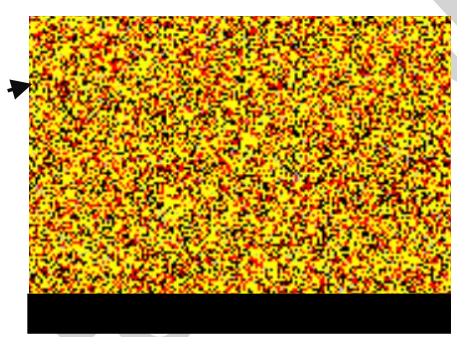

1 week

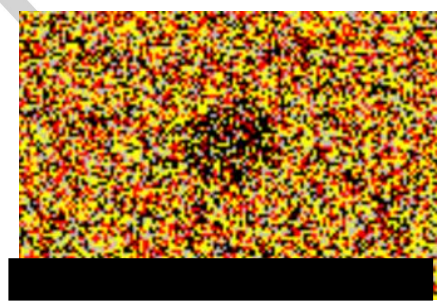

12 weeks

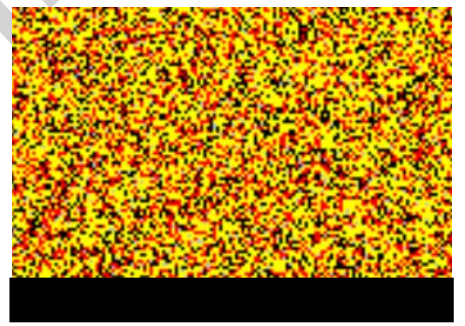

2 weeks

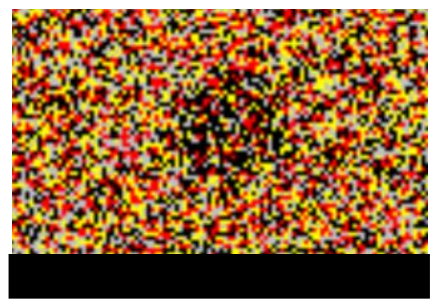

24 weeks

Fig.10. The cellular strength phase evolution of the degradation process of the PLA device with initial crystallinity in Ref.[27] (case 3(b)). Yellow represents the amorphous strength phase. Red represents the crystalline strength phase. Grey represents one kind of the strength vacancy phase which the cellular status is the amorphous $\operatorname{status}(x(i, j, t)=1$ or $x(i, j, t)=0)$ but its molecular 


\section{ACCEPTED MANUSCRIPT}

weight is smaller than $M_{n c}^{\sigma}$. Black represents another kind of the strength vacancy phase with hole cell status $(x(i, j, t)=-1)$.

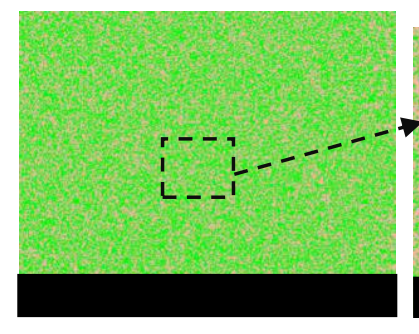

0 week

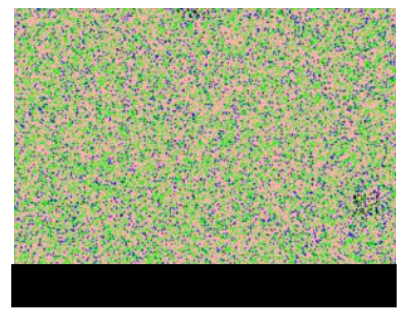

8 weeks

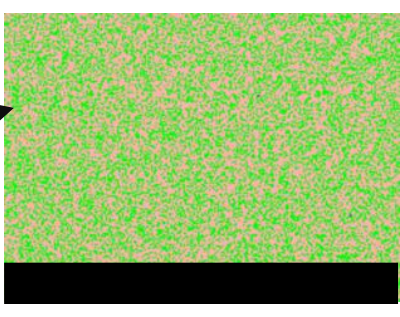

1 week

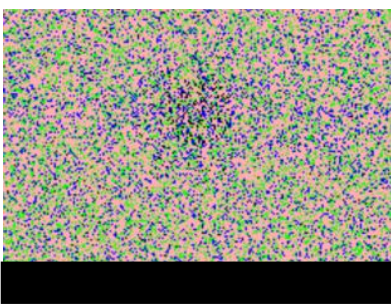

12 weeks

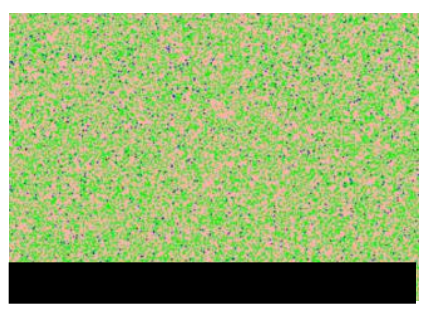

2 weeks

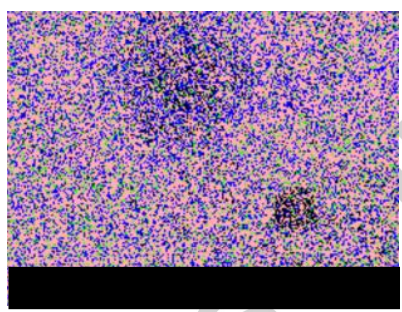

24 weeks

Fig.11. The cellular status evolution of the degradation process of PLA device with initial crystallinity in Ref.[27]( case 3(b)). Green stands for the initial amorphous status $(x(i, j, t)=1)$. Pink stands for the cells with crystallization status $(x(i, j, t)=-2)$. Blue stands for the cells with chain scission reaction $(x(i, j, t)=0)$. Black stands for the cells with holes in them $(x(i, j, t)=-1)$.

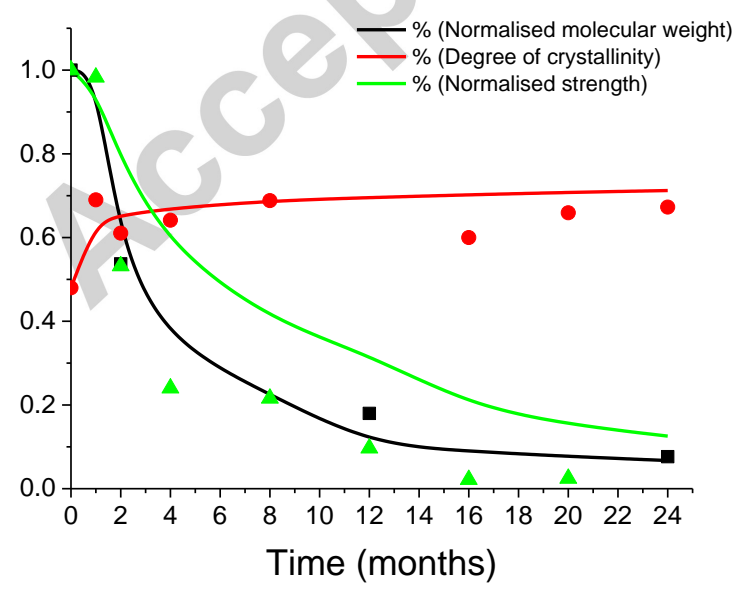

Fig.12. Comparison between the multiscale heterogeneous strength model and the experimental data of sample with initial crystallinity (case 3(b)) obtained by Duek et al. [27] for number 


\section{ACCEPTED MANUSCRIPT}

average molecular weight, volume degree of crystallinity and strength as functions of degradation time for the polymer sample. The continuous lines represent the model prediction while the discrete symbols are the experimental data.

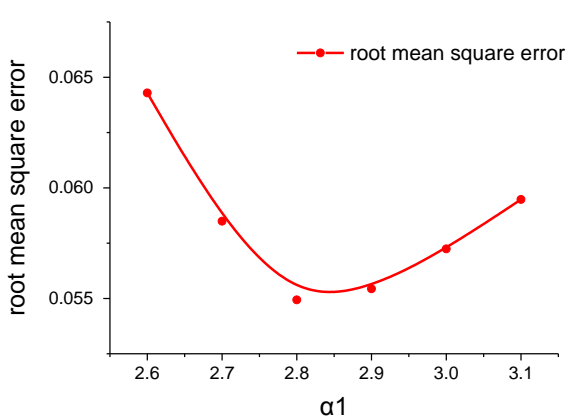

(a)

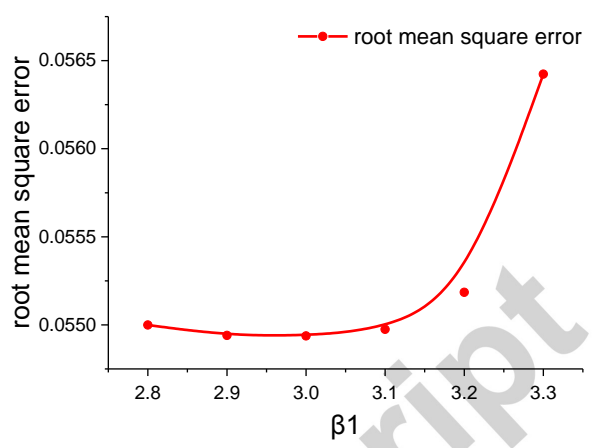

(b)

Fig. 13. The analysis of root mean square error (rmse) for normalized strength with the change of model parameter $\alpha_{1}$ and $\beta_{1}$, the experimental data is from Ref. [24] and the calculation values is by multi-scale heterogeneous strength model.

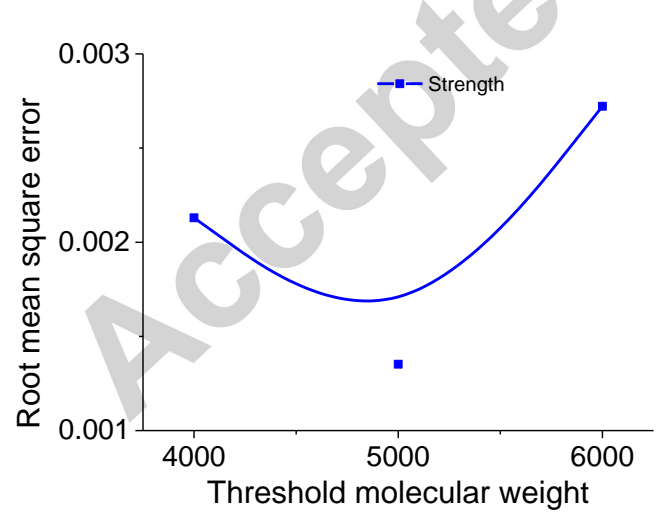

Fig.14. The analysis of root mean square error (rmse) for normalized strength with the change of model prameter $M_{n c}^{\sigma}$, the experimental data is from Ref. [26] and the calculation values is by multi-scale heterogeneous strength model. 


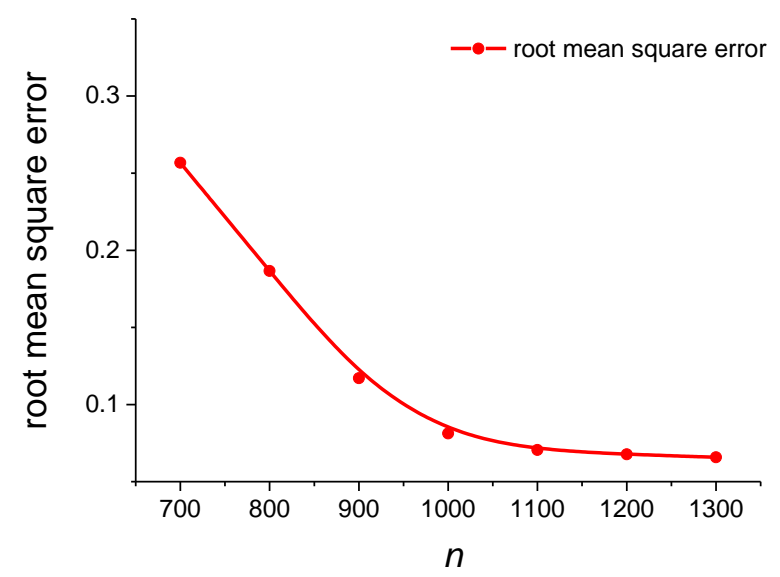

Fig. 15 The analysis of root mean square error (rmse) for normalized average molecular weight with the change of model parameter $n$, the experimental data is from Ref.[24].

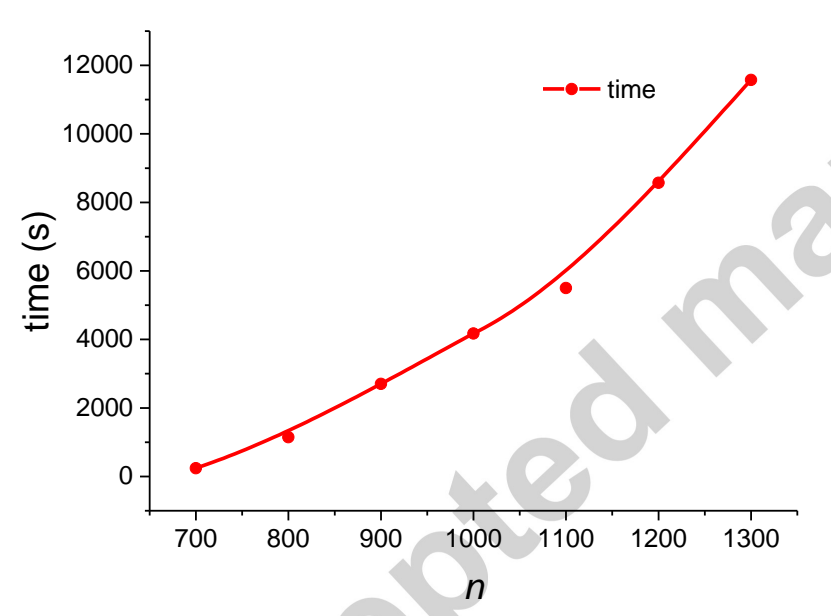

Fig. 16 The analysis of computation time of the strength model with different values of model parameter $n$, Case 1[24] is as the example. 


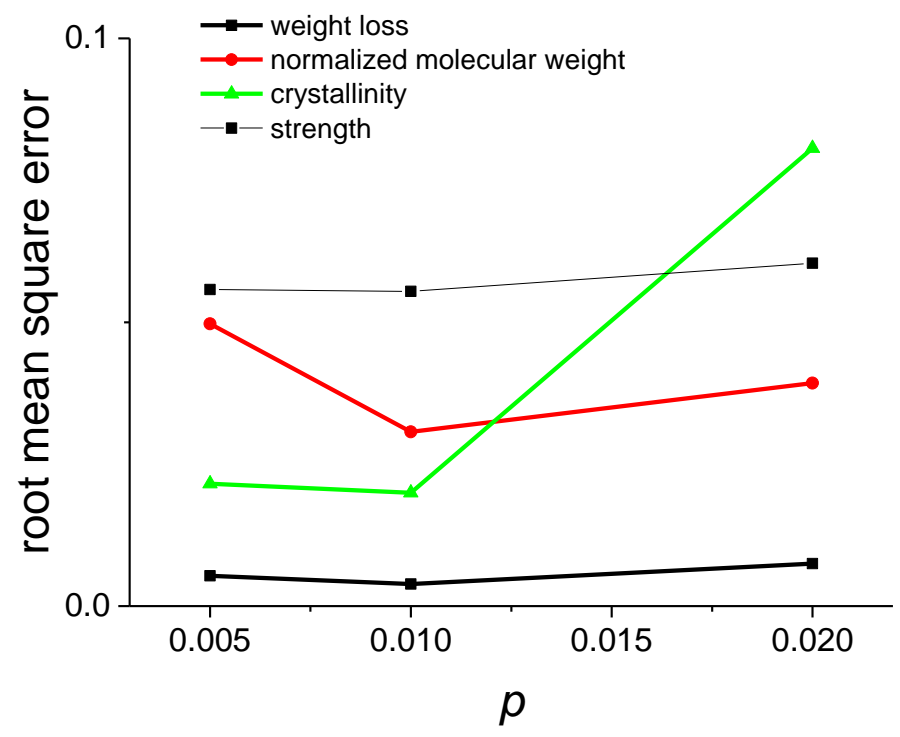

Figure 17. The analysis of root mean square error (rmse) for normalized average molecular weight, weight loss, crystallinity, strength with different values of model parameter $p$, the experimental data is from Case 2 (Ref. [26]).

Table 1- Model parameters that provide the best fit between the heterogeneous strength model and the experimental data of three calculation cases.

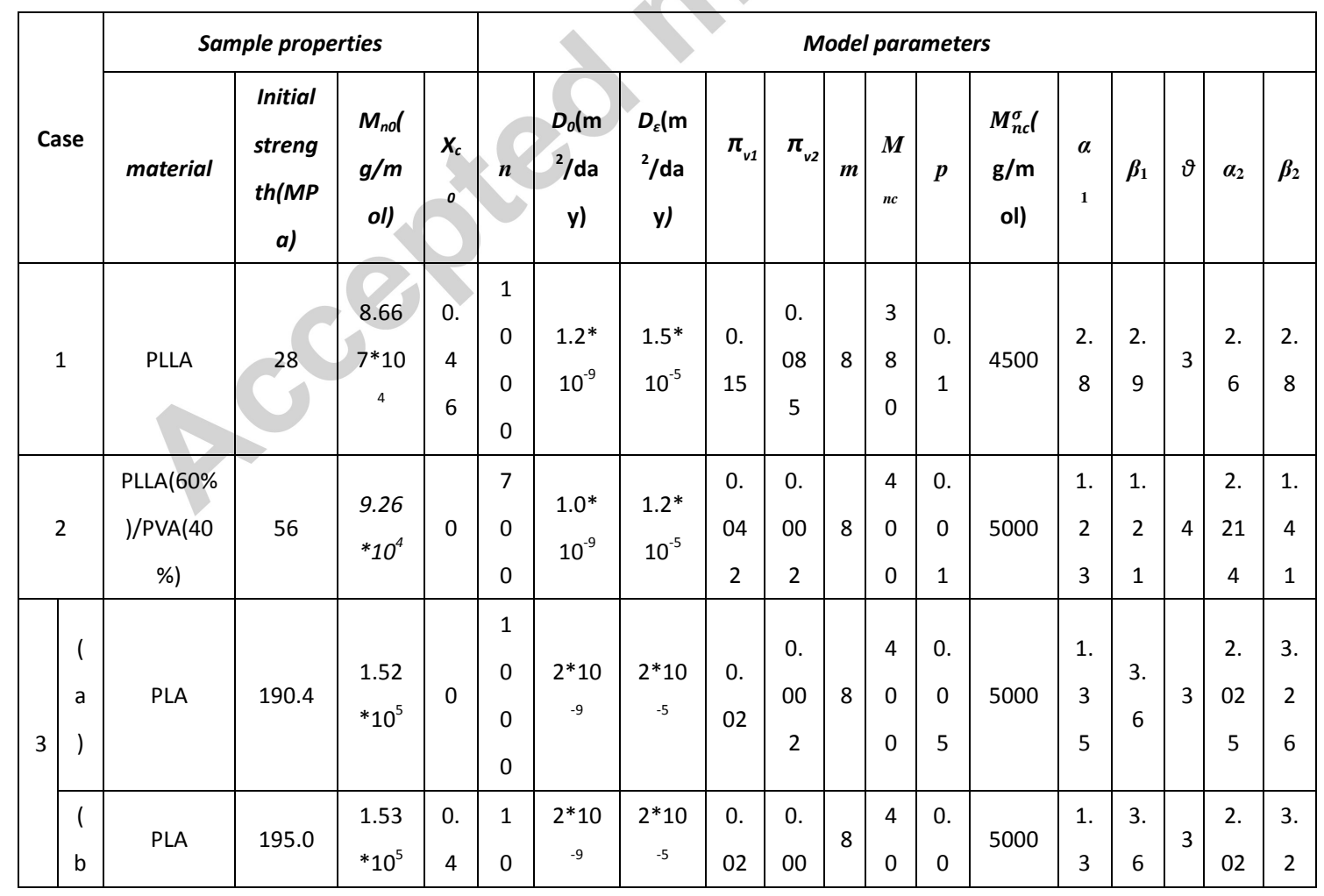




\begin{tabular}{|l|l|l|l|l|l|l|l|l|l|l|l|l|l|l|l|l|l|l|}
\hline 1 & & & & 8 & 0 & & & & 2 & & 0 & 1 & & 5 & & & 5 & 6 \\
\hline
\end{tabular}

